\title{
Motivation Mediates the Influence of the Knowledge of Nutrients' Function on Diet
}

\author{
Michael Lindbloom¹, Jebaraj Asirvatham, ", Wanki Moon², Ira Altman² \\ ${ }^{1}$ Elmhurst College in Elmhurst, Illinois, the United States \\ ${ }^{2}$ Department of Agribusiness Economics, Southern Illinois University Carbondale, Illinois, the United States \\ Email address: \\ michaellindbloom@gmail.com (M. Lindbloom), jebaraj@siu.edu (J. Asirvatham),wmoon@siu.edu (W. Moon), \\ ialtman@siu.edu (I. Altman) \\ ${ }^{*}$ Corresponding author
}

\section{To cite this article:}

Michael Lindbloom, Jebaraj Asirvatham, Wanki Moon, Ira Altman. Motivation Mediates the Influence of the Knowledge of Nutrients' Function on Diet. Journal of Food and Nutrition Sciences. Vol. 9, No. 1, 2021, pp. 1-9. doi: 10.11648/j.jfns.20210901.11

Received: December 9, 2020; Accepted: January 5, 2021; Published: January 18, 2021

\begin{abstract}
Unhealthful diets are strongly linked to health problems, including obesity, cancer, heart disease, and diabetes. Past efforts to improve population diet centered on conveying the importance of nutrition and nutrition knowledge. This study examines if the effect of the knowledge of specific nutrient's function on dietary behavior is mediated by motivation towards better health. Survey data used in this study was obtained via a web-panel survey that was conducted by Ipsos-Observer. We employed the mediation analysis method that is established in the literature. We find that motivation mediates the influence of the knowledge of nutrients' function on dietary behaviors. Individuals with high health-motivation and higher levels of the knowledge of nutrients' dietary functions exhibit healthier dietary behaviors than individuals with low-health motivation but high levels of nutrients' functional knowledge. Furthermore, individuals with health problems exhibited higher motivation to maintain healthy diet. A key implication is that efforts to promote healthy dietary behaviors should consider motivation in addition to providing tools, such as nutrients' functional knowledge and nutrition labels. Simply conveying the importance of nutrition itself may not be enough to motivate people to affect diet changes. Educating about the specific role of nutrients' in human health might motivate more individuals to modify diet.
\end{abstract}

Keywords: Diet Behavior, Nutrients' Dietary Function, Motivation, Nutrition, Diet

\section{Introduction}

Diet is one of the most critical components for maintaining health, and as such, unhealthful diets are strongly linked to health problems, including obesity, cancer, heart disease, and diabetes [1-3]. There have been various efforts by governmental and non-governmental institutions and organizations [4-6]. Efforts include education, motivation through apps, faith-based, and others. Previous research has found that unhealthy dietary behavior is commonly associated with several factors, including nutritional knowledge and demographic variables [7]. Along with knowledge and personal characteristics, there are studies showing that another variable, health motivation, also plays an important role in healthy eating [8-10]. For example, Moorman and Matulich [11] show that health motivation moderates the effect of health ability on preventive health behaviors as well as directly affecting it. In a study on the use of nutrition facts panel (NFP) on making healthy choices, Miller and Cassady [12] found that both knowledge and motivation are important and that knowledge plays a mediating role for motivation. Michaelidou et al. [13] show that intrinsic motives for health optimization are the strongest predictor of healthy eating behavior for UK adult consumers. In addition, Sun et al. [14] present evidence of health motivation mediating the effects of personal characteristics on a healthy diet of US and Chinese college students. A better understanding of what motivates an individual to consume a healthy diet and its relationship with health-specific nutrient knowledge could provide a firm foundation for our understanding of how people make dietary choices. Past literature have noted the importance of motivation on dietary behavior in the context of nutrition knowledge but has not 
studied it in the context of the knowledge of nutrients' dietary functions using mediation analysis. This study contributes to this literature.

Building on these studies, the purpose of this study is to empirically estimate the role of motivation in maintaining dietary health among residents in the United States. Even though there is research on motivation and diet, no study, to the best of our knowledge, has formally tested whether motivation mediates healthy dietary choices. Conceptually, for an individual to consume a healthy diet he or she must be: (a) knowledgeable about the role specific nutrients play in maintaining health; (b) motivated to maintain a healthy diet (or to improve a poor diet); and (c) for nutritional knowledge to have an effect on dietary choices, the individual must also be motivated to pursue a healthy diet. The primary hypothesis being tested in this article is that while the knowledge of nutrients' dietary functions and certain demographic characteristics have predictable and measurable effects on dietary behavior, these effects are mediated by dietary health motivation. Path analysis is employed to determine whether the effect of health-specific nutrient knowledge on dietary behavior is mediated by motivation.

Motivation to consume a healthy diet could be influenced by a number of factors, including education, social network, current health status, personal aspirations, health concerns and the decisions of the household food purchaser $[9,15]$. Measuring motivation, however, is challenging because it is not easily observable. This study utilizes qualitative consumer survey response data to construct an empirical measurement of motivation. The consumer survey was administered by a national polling corporation (IpsosObserver).

\section{Motivation: A Key Factor on Dietary Behavior}

A general definition of a healthy diet, according to many dietitians, clinical nutritionists, and government agencies, including US Department of Agriculture (USDA), Centers for Disease Control and Prevention (CDC), US Department of Health and Human Services (HHS), is one that helps to maintain or improve health. More specifically, a healthy diet is one that balances micronutrients, macronutrients and calories in a way that maximizes resistance to disease and maintains a healthy weight. The public, however, understands healthy and unhealthy eating in several different ways [16]. For example, responses to their open-ended questions about what constitutes a healthy diet included: eating food containing fiber, eating natural foods, eating food containing vitamins, eating fresh foods, avoiding fried foods, and eating a variety of food. Similarly, an array of responses was recorded for open-ended questions concerning unhealthy diets. These included: eating foods with high-fat content and high sugar content, eating fried foods, eating food with additives, and eating manufactured/processed foods. Other studies report similar findings [17] after recording answers to open-ended questions about healthy and unhealthy diets. Despite the variation in answers, public conceptions on a healthy or unhealthy diet generally align with the definition outlined by professional dietitians and clinical nutritionists, indicating that even a cursory understanding of dietary health would promote or enable healthy dietary decisions.

Similar to studies on knowledge of dietary health, studies from the past several decades have found that dietary motivation is also defined and understood in a variety of ways. For example, in 1952, Hochbaum, Rosenstock and Kegels [18] developed the Health Belief Model, which combined social, psychological and behavioral theories in an effort to explain and predict individuals' health behaviors. The Health Belief Model has been used as a framework to map behavior, particularly on health topics. For example, Becker et al. [19] used this framework for understanding mothers' adherence to a dietary regime for their obese children. The original model posits "disease avoidance" as the primary incentive for acting in ways to enhance health. In other words, a high fear arousal in the parents of obese children resulted in the most significant weight loss compared to the control group, who experienced weight gain. This implies that simply informing parents or individuals at risk of health problems linked to the diet might not inspire a change in diet, but a different form of motivating factor (i.e., fear arousal, probability of illness occurring, or positive motivating factors) might be necessary to initiate long-term transformations.

Eikenberry and Smith [17] studied motivational and prohibitive factors associated with healthy dietary behavior and found links to demographic variables. For example, there were noticeable differences in motivation and prohibitive factors across races and income levels. Higher income respondents generally placed more importance on "health," whereas lower income respondents wanted to "look good." In terms of race, Caucasian respondents wanted to "feel good," whereas African-American respondents wanted to "live longer."

A more recent work shows different motivational factors among vegetarians and the low and medium meat eaters [9]. Vegetarians cited taste- and animal-welfare related reasons, while the low and medium meat-eaters indicated health as the primary reason. This carries relevance to policies, particularly those that seek to influence dietary choices. The results of most of these studies support the notion that people can be motivated in different ways to eat healthier. Still, those motivations can mediate or be mediated by social demographics.

The theoretical foundation in the current study is that motivation and health-specific nutrient knowledge are essential components for maintaining healthful dietary behaviors. More specifically, individuals with higher levels of health motivation will have healthier dietary behaviors than individuals with lower levels of health motivation. Likewise, nutrients' functional knowledge is theorized to be positively correlated with healthy dietary behavior. However, this positive effect of health-specific nutrients' knowledge on 
dietary behavior is hypothesized to be mediated by motivation. Thus, respondents with high levels of both motivation and health-specific nutrients' knowledge will exhibit higher levels of healthy dietary behaviors than individuals with low levels of health motivation and high levels of the knowledge of nutrients' dietary functions. In order to measure this mediating role of motivational factors on dietary behaviors, path analysis concepts are applied to household survey data collected in the United States in 2008.

\section{Data}

The data for this project was obtained from a web-panel survey conducted by Ipsos-Observer. Ipsos-Observer is a private consulting firm that specializes in conducting consumer research and public opinion polls on food-related topics. This particular survey drew 3,456 responses from a stratified sample of 9,000 households (38.4\% response rate). The survey asked questions concerned with the general health status, dietary health motivations, dietary behaviors and knowledge of nutrients' dietary functions, as well as several key demographic variables relevant to this study (age, gender, income, and education level). Indices of motivation, knowledge, and dietary behavior were created using the survey question items listed in Table 1. The question items were drawn from Moorman and Matulich [11]. Given the multiple items used to construct such indices, internal consistency was tested using Cronbach alphas. Table 2 presents the Cronbach's alphas along with summary statistics for the variables used in this article. The value for Motivation was 0.798 and for Behavior was 0.867 , suggesting that the two measures were internally consistent and adequate enough to complete the path analysis.

Table 1. Questions used for operationalization of variable indices.

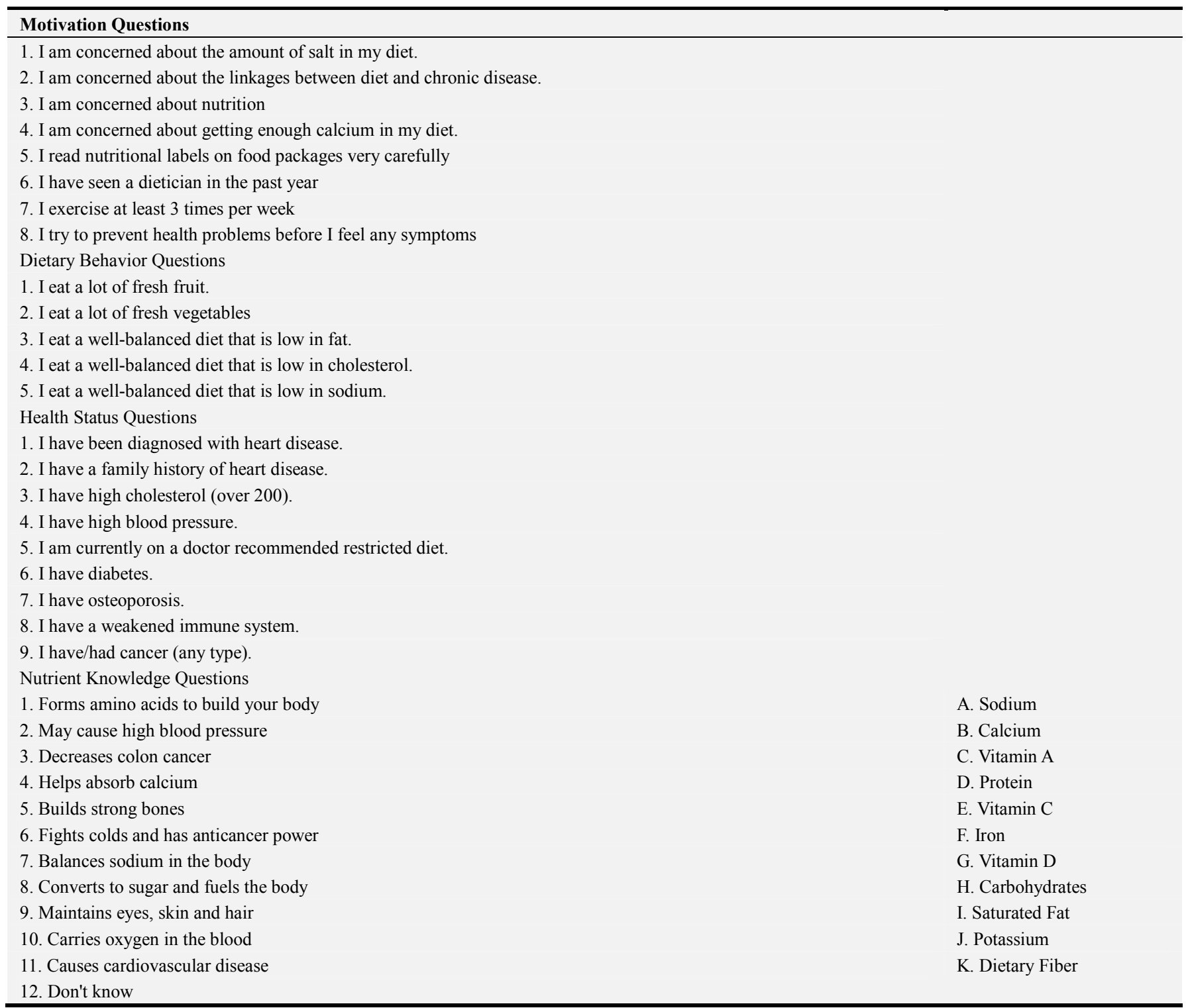


Table 2. Descriptive statistics of variables used in regression analyses.

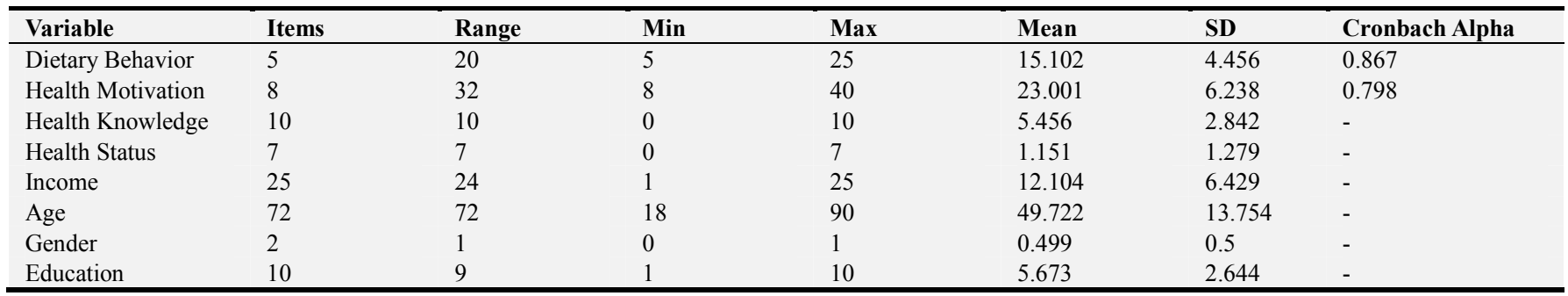

In order to quantify the latent variable "Motivation," responses to a series of Likert-scale questions were pooled. The word 'concern' is used in half of the questions, and 'concern' is implied in the other half. These responses were used because it is assumed that if the individual is concerned about the particular health or diet issue then this could also indicate that the respondent has both the intention and desire to achieve a healthful state of being. The definition of concern is "something that interests you because it is important or affects you." Therefore, if a person is 'concerned' or interested about the healthfulness of their diet, he or she is expected to be motivated to act upon this concern. The questions which do not specifically use the word 'concern' intuitively imply 'concern.' For example, if a person reads nutrition labels, it is expected that he or she is 'concerned' about the ingredients and nutrient make-up of the food at hand. If a person has seen a dietician in the past year, he or she is 'concerned' about dietary choices. The presence of an individual's 'concern' in this regard indicates a psychological stimulus which in turn would most likely affect the respondent's behavior. Given that the response to each statement ranges from 1 to 5 , the sum of the response values was used as an index to classify the motivation variable. The index is then treated as a continuous explanatory variable, with higher values indicating higher levels of motivation. Because there are eight questions, this variable can range in value from 8 to 40 .

Next, to quantify a respondent's actual dietary behavior, survey responses were again indexed. This time the questions were specifically concerned with the respondents' dietary behaviors (Table 1). Given that there are five questions, this variable could potentially range in value from 5 to 25 . Here again, the higher the sum the healthier the dietary behaviors of the respondent.

In order to measure an individual's level of health-specific nutrients' knowledge, the respondent was presented with two lists: (1) a list of nutrients and (2) a list of nutrient roles in the human body (Table 1). The respondent was then tasked with matching the nutrient to its role in the body. For example, for nutrient potassium, the correct matching role is that it helps the body absorb calcium. According to the National Institutes of Health, Office of Dietary Supplements, a diet with too little potassium can deplete calcium in bones, whereas high intakes of potassium seem to result in stronger bones and bone mineral density [20]. The respondent then has the option to guess the correct response or utilize the response "I don't know." The number of correct responses was summed, and this sum was used as the continuous health knowledge variable (with higher totals being associated with higher levels of nutrients' dietary functional knowledge). ${ }^{1}$

Finally, to quantify health status, nine questions were asked and each 'yes' answer was summed to create a total. Higher numbers for this variable means a less healthy individual because these are detrimental health conditions. Although these health status questions are not specifically about the individual's diet, each health condition is highly correlated to an unhealthy diet. For example, one of the health conditions is high cholesterol. The Mayo Clinic, one among few websites that provide reliable medical info, lists several factors causing this condition, with three of them being poor diet, obesity, and large waist circumference [21]. Another health condition is high blood pressure, an infliction, according to Mayo clinic posts, can be controlled through reduced weight, eating a healthy diet, and reducing the amount of sodium in the diet, among other proactive activities.

Demographic variables in the regression models include age, gender, income, and level of schooling. Age is included as a continuous explanatory variable, whereas gender is a binary variable ( 1 for male, 0 for female). Income is grouped into 25 categories and is also included as a continuous variable. The itemization of the categories is shown in Table A1 in the Appendix. For example, if the respondent's income is between $\$ 50,000$ - $\$ 54,999$, then the income variable is 11 . Because the group number increases with income levels, these group numbers are used as a continuous variable. Finally, level of schooling is categorized into 10 groups and used as a continuous variable (Table A2 in the Appendix). For example, if the respondent has a four-year college degree, then the category number of level of schooling is 6 .

Summary statistics for these variables are provided in Table 2. The values of the indices for dietary behavior show that respondents on average consumed neither an entirely healthy nor an entirely unhealthy diet. The average score for dietary behavior is 15.102 , with a standard deviation of 4.456 (ranging from 5 to 25). In regards to the motivation variable, the mean value is 23.001 , with a standard deviation of 6.238 . This variable can range in value from 8 to 40 , so similar to the dietary behavior index, the average for the motivation variable is also somewhat in the middle of potential values. For the health knowledge index, the max value is 10 and the

1 Note that if the respondent answered "I Don't Know," this was recorded as an incorrect answer and given a value of zero. 
minimum value is 0 , and the mean value was 5.456 ( $\mathrm{SD}$ of 2.842). This indicates that respondents on average answered about half of the questions correctly.

Regarding gender, females comprised half of the respondents and in terms of income, the average category score is 12.104 which translates to an income level in the range of $\$ 55,000-\$ 59,999$. This range falls very close to the average household income seen nationally in the United States, indicating a fairly representative group of respondents Finally, the mean value of the education variable is 5.673, with a standard deviation of 2.644. This mean value corresponds to an education level that is equivalent to graduating from a 2-year college or completing a technical or vocational school. Again, this seems to be fairly representative of the general population in the United States.

\section{Empirical Model Specification}

The main objective of this study is to examine if health motivation mediates the effect of the knowledge of nutrients' dietary function on dietary behavior. Empirical models are specified according to the procedures proposed by Baron and Kenny [22]. Kenny, Kashy and Bolger [23] further elaborate on the originally proposed four-steps. Applying BK's mediation hypothesis on the dietary-motivation context, it is hereby propose that the following four conditions should be met for the mediation hypothesis to hold: (i) motivation should have a significant effect on dietary behavior; (ii) knowledge of nutrients' dietary functions should have a significant effect on dietary behavior; (iii) nutrients' functional knowledge should have a significant effect on health motivation; and (iv) when both motivation and knowledge are included in the regression model of dietary behavior as the dependent variable, the effect of knowledge on dietary behavior becomes insignificant (complete mediation) or becomes smaller (partial mediation). At first, the Behavior variable $\left(B e h_{i}\right)$ is regressed on each of the variables Motivation $\left(\right.$ Mot $\left._{i}\right)$ and Knowledge $\left(\right.$ Know $\left._{i}\right)$ in separate regressions (Equations 1 and 2, respectively). In Equation 3, both variables are included in the same model. This assesses the significance of these variables in explaining dietary behavior in isolation and together. Carrying on, Motivation is regressed on Knowledge to validate the condition (c), Equation 4 below. The results of the following four models are provided in Table 3. Equations 1 through 4 are:

$$
\begin{aligned}
& \text { Beh }_{i}=\beta_{0}+\beta_{1} \text { Mot }_{i}+\varepsilon_{1 i} \\
& \text { Beh }_{i}=\beta_{0}+\beta_{1} \text { Know }_{i}+\varepsilon_{2 i} \\
& \text { Beh }_{i}=\beta_{0}+\beta_{1} \text { Mot }_{i}+\beta_{2} \text { Know }_{i}+\varepsilon_{3 i} \\
& \text { Mot }_{i}=\beta_{0}+\beta_{1} \text { Know }_{i}+\varepsilon_{4 i}
\end{aligned}
$$

The next five equations include control variables, such as Health Status, and demographic characteristics, including Income, Age, Education Level and Gender. The model specifications are thus:

$$
\begin{aligned}
& B e h_{i}=\beta_{0}+\beta_{1} H S_{i}+\beta_{2} \operatorname{Inc}_{i}+\beta_{3} A g e_{i}+\beta_{4} E d u_{i}+\beta_{5} G_{i}+\varepsilon_{5 i} \\
& \text { Beh }_{i}=\beta_{0}+\beta_{1} \text { Mot }_{i}+\beta_{2} \text { Know }_{i}+\beta_{3} H S_{i}+\beta_{4} \operatorname{Inc}_{i}+\beta_{5} A g e_{i}+\beta_{6} E d u_{i}+\beta_{7} G_{i}+\varepsilon_{6 i} \\
& \mathrm{Beh}_{i}=\beta_{0}+\beta_{1} \text { Know }_{i}+\beta_{2} H S_{i}+\beta_{3} \operatorname{Inc}_{i}+\beta_{4} \text { Age }_{i}+\beta_{5} E d u_{i}+\beta_{6} G_{i}+\varepsilon_{7 i} \\
& \mathrm{Beh}_{i}=\beta_{0}+\beta_{1} \operatorname{Mot}_{i}+\beta_{2} H S_{i}+\beta_{3} \operatorname{Inc}_{i}+\beta_{4} A g e_{i}+\beta_{5} E d u_{i}+\beta_{6} G_{i}+\varepsilon_{8 i} \\
& \text { Mot }_{i}=\beta_{0}+\beta_{1} \text { Know }_{i}+\beta_{2} H S_{i}+\beta_{3} I n c_{i}+\beta_{4} A g e_{i}+\beta_{5} E d u_{i}+\beta_{6} G_{i}+\varepsilon_{9 i}
\end{aligned}
$$

Where: $H S_{i}$ refers to the health status of individual $i, \operatorname{Inc}$ refers to income level, Age is of course the age of individual $i, E d u_{i}$ is the education level of the individual, and finally $G_{i}$ is the dummy control variable for gender, with 0 representing women and 1 representing men. Results for equations 5 through 9 are listed in Table 4.

\section{Estimation Results}

The value of the coefficient for motivation (0.543) for equation 1 as well as its t-statistic (68.78) indicate a significant and positive effect of the motivation index on dietary behavior (Table 3). Additionally, the results for Equation 2 indicate a positive (0.2601) and significant (9.89) relationship between Knowledge and behavior. However, when combining Motivation and Knowledge together on the right-hand side, the coefficient on the Knowledge variable (0.019) not only decreases in magnitude but also becomes statistically insignificant (1.12) in explaining dietary health behaviors. This specific type of change in coefficient values and t-statistics is a necessary result to show that the effect of the knowledge of nutrients' dietary functions on dietary behavior is mediated by motivation.

Table 3. Parameter Coefficient Estimates for Regression Equations 1-4.

\begin{tabular}{lllll}
\hline \multicolumn{5}{c}{ Dependent Variable } \\
\cline { 2 - 5 } & Behavior & Behavior & Behavior & Motivation \\
\hline $\begin{array}{l}\text { Equation Number } \\
\text { Variables }\end{array}$ & 1 & 2 & 3 & 4 \\
Intercept & 2.611 & 13.683 & 2.545 & 20.579 \\
& $(13.88)^{*}$ & $(84.56)$ & $(12.91)$ & $(91.48)$ \\
Motivation & 0.5431 & - & 0.541 & - \\
& $(68.78)$ & - & $(67.13)$ & - \\
Knowledge & - & 0.2601 & 0.019 & 0.444 \\
& - & $(9.89)$ & $(1.12)$ & $(12.14)$ \\
R-squared & 0.578 & 0.027 & 0.578 & 0.041 \\
\hline
\end{tabular}

*t-statistics are reported in parenthesis.

As the second step in showing that nutrients' dietary functional knowledge is mediated by motivation, a regression 
of Motivation on Knowledge was computed with no controls (Equation 4), and the estimates are positive (0.444) and statistically significant (12.14) relationship. The results of this regression are thus in favor of the hypothesis that Motivation has a mediating effect on Knowledge. That is, individuals with high levels of nutrients' dietary functional knowledge and high levels of positive dietary motivation will engage in healthier dietary behaviors than individuals with high levels of dietary nutrients' dietary functional knowledge and low levels of positive dietary motivation. The results suggest that a person who is knowledgeable about the components of a healthy diet will only exhibit healthy dietary behaviors if that individual is motivated to do so.
In the next step, more control variables are added. Table 4 presents estimates from the remaining five model specifications, Equations 5 through 9. Equation 5 results show that the coefficients and significance levels for the newly added demographic variables were all statistically significant in explaining dietary behavior. All coefficients were positive except for gender. The negative gender coefficient indicates that women engage in healthier dietary behaviors than men (recall that women are coded as 0 , and men as 1. Health Status is significant with a positive sign, which shows that healthier individuals engage in a less healthy dietary behavior.

Table 4. Parameter Coefficient Estimates for Regression Equations 5-9.

\begin{tabular}{|c|c|c|c|c|c|}
\hline & \multicolumn{5}{|c|}{ Dependent Variable } \\
\hline & Behavior & Behavior & Behavior & Behavior & Motivation \\
\hline Equation Number & 5 & 6 & 7 & 8 & 9 \\
\hline \multicolumn{6}{|l|}{ Variables } \\
\hline \multirow[t]{2}{*}{ Intercept } & 10.70 & 0.589 & 10.182 & 0.547 & 18.061 \\
\hline & (33.29)* & $(2.23)$ & (30.65) & $(2.09$ & (38.69) \\
\hline \multirow[t]{2}{*}{ Motivation } & - & 0.531 & - & 0.529 & - \\
\hline & - & (65.86) & - & $(66.42$ & - \\
\hline \multirow[t]{2}{*}{ Knowledge } & - & -0.021 & 0.157 & - & 0.336 \\
\hline & - & $(-1.16)$ & $(5.82)$ & - & $(8.84)$ \\
\hline \multirow[t]{2}{*}{ Health Status } & 0.249 & -0.111 & 0.227 & -0.113 & 0.635 \\
\hline & $(4.04)$ & $(-2.69)$ & (3.69) & $(-2.74)$ & (7.36) \\
\hline \multirow[t]{2}{*}{ Income } & 0.039 & 0.022 & 0.032 & 0.021 & 0.019 \\
\hline & $(3.06)$ & $(2.57)$ & $(2.50)$ & $(2.48)$ & $(1.05)$ \\
\hline \multirow[t]{2}{*}{ Age } & 0.051 & 0.036 & 0.050 & 0.036 & 0.027 \\
\hline & $(8.92)$ & $(9.43)$ & $(8.78)$ & $(9.41)$ & $(3.34)$ \\
\hline \multirow[t]{2}{*}{ Education Level } & 0.278 & 0.104 & 0.234 & 0.098 & 0.244 \\
\hline & $(8.75)$ & $(4.76)$ & (7.19) & $(4.63)$ & $(5.35)$ \\
\hline \multirow[t]{2}{*}{ Gender } & -0.944 & -0.190 & -0.797 & -0.173 & -1.142 \\
\hline & $(-6.18)$ & $(-1.85)$ & $(-5.17)$ & $(-1.70)$ & $(-5.27)$ \\
\hline R-Squared & 0.076 & 0.595 & 0.085 & 0.594 & 0.078 \\
\hline
\end{tabular}

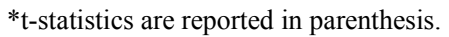

Equation 6 adds motivation and knowledge to the righthand side, which provides some interesting results. For example, as shown in Table 3, the magnitude of the Health Status coefficient $(-0.111)$ and t-statistic $(-2.69)$ did not change significantly. However, the negative sign implies that individuals with a healthier state of being will engage in healthier dietary behaviors when motivation and knowledge are accounted for. This implies that the positive sign of Health Status on Behavior is due to the absence of Motivation. In other words, when Motivation is included, the positive dietary effects are embedded in Motivation and the net effect of Health Status becomes negative. Moorman and Matulich [11] did find that higher perceived health status levels corresponded to healthier behaviors when motivation was high rather than low. Given that the health status variable is somewhat stated and perceived, this could be an area further investigation.
The Gender coefficient (-0.190) and t-statistic (-1.85), did change substantially so that it is no longer a statistically significant when motivation was included in the model. This is suggestive of a mediation effect. The changes in the remaining variables' coefficients are not considerable, which lend to the notion that they either affect dietary behavior independently or have a marginal correlation with other variables. Also worth noting from the results of equation six, however, is the negative sign of the Knowledge variable. This change in sign from equation three could imply that Knowledge might have some interaction effects with some of the demographic variables. However, because the statistical significance level has not changed and no substantial correlation among variables was observed, one can safely ignore the sign change. Correlation among variables is reported in Table 5 . 
Table 5. Correlation Matrix for Variables Used in Regression Analyses.

\begin{tabular}{|c|c|c|c|c|c|c|c|}
\hline & Motivation & Status & Knowledge & Income & Age & Education level & Gender \\
\hline Motivation & 1 & & & & & & \\
\hline Health Status & 0.1930 & 1 & & & & & \\
\hline Knowledge & 0.2054 & 0.0399 & 1 & & & & \\
\hline Income & 0.0655 & -0.0837 & 0.2195 & 1 & & & \\
\hline Age & 0.1327 & 0.3173 & 0.0751 & 0.0419 & 1 & & \\
\hline Education Level & 0.0957 & -0.0290 & 0.2265 & 0.3426 & 0.0375 & 1 & \\
\hline Gender & -0.0750 & 0.0614 & -0.0920 & -0.0237 & 0.0905 & 0.2457 & 1 \\
\hline
\end{tabular}

Moving forward, Equation 7 excludes motivation from the right-hand side but includes knowledge. Equation 8, on the other hand, includes motivation on the right-hand side but excludes knowledge. The impact of the demographic characteristics on Motivation becomes much clearer upon examination of these results. For example, when Motivation is included as an independent variable (equation 8) as opposed to Knowledge (equation 7), the Gender coefficient becomes statistically insignificant and its magnitude is substantially reduced.

The final step in testing for mediation is to regress the mediator (Motivation) on Knowledge and the demographic variables (Equation 9). The results confirm the mediation effects of Motivation on Gender and Knowledge, as well as the partial mediation of Motivation on Health Status. Notice that the coefficient for Health Status is 0.635 and its corresponding t-statistic is 7.36. Because Health Status was statistically significant in equations 5 and 6 and that this magnitude increased in equation 9 , it can be affirmed that there is a partial mediation effect. The remaining variables do not show any sign of mediation. This is particularly obvious with the Income variable, which has a more independent effect on Behavior.

\section{Discussion, Conclusions, and Implications}

The purpose of the regression analysis in this study was to better understand the effects that motivation, nutrients' dietary functional knowledge, and demographic variables have on dietary behaviors. It was hypothesized that individuals with higher levels of health motivation will exhibit higher levels of healthy dietary behaviors than individuals with lower levels of health motivation. The results of regression equations 1 and 3 (as displayed in Table 3) do in fact validate this hypothesis. The positive relationship that exists between Behavior and Motivation indicates that higher levels of health motivation correspond to higher levels of healthy dietary behavior in a statistically significant manner. Similarly, nutrients' dietary functional knowledge is shown to have a positive and statistically significant relationship with dietary behavior, as shown by the results of regression Equation 2.

Despite the statistically significant coefficients for Motivation and Knowledge in equations 1 and 2 however, there is a considerable difference in the R-squared values. All of the regression models that included motivation resulted in a high R-square of at least 0.578 . Whereas R-square in regression models without Motivation was lower than 0.08 . This indicates that given the responses to this survey, Motivation has a much stronger effect on dietary behavior when compared to Knowledge. These differences R-square values also support the notion that motivation to engage in healthy dietary behavior is a more accurate determinant of dietary behavior than nutrients' dietary functional knowledge.

One issue that needs to be addressed of course is the way in which the Behavior, Motivation and Knowledge variables were constructed. First, the Behavior variable is a stated variable rather than a revealed variable. This means that dietary behaviors of respondents are not tracked by some external source, but are instead declared by the respondent. This could potentially lead to a survey response bias. Similar to the stated behaviors, Motivation is measured by respondents' stated response to survey questions. This could result in a survey response bias because a person could actually be less or more motivated than what the survey questions reveal. This, however, is a constraint to most survey studies.

Also worth mentioning is that the index of the knowledge of nutrients' dietary functions was constructed by testing for knowledge. In this case, either the respondent knows the corresponding health effect of the listed nutrient, or he does not. This could lead to contrasting levels of motivation and knowledge, because as Povey, and Eikenberry and Smith [16] found, much of the public have basic ideas about how to consume a healthy diet but lack in specific nutrients' dietary functional knowledge. Therefore, for example, an individual may be motivated to eat a diet comprised primarily of fruits and vegetables, but be unaware of the specific nutritional value gained from such a diet.

The second part of the hypothesis stated that individuals with higher levels of motivation and higher levels of the knowledge of nutrients' dietary functions would exhibit higher levels of healthy dietary behaviors than individuals with lower levels of health motivation and high levels of nutrients' dietary functional knowledge. This was also confirmed by the analysis. Results in Tables 3 and 4 show that the coefficient for the Motivation variable was consistent across different specifications and highly statistically significant in all (t-statistics were 68.78 and 67.13), whereas the Knowledge variable was significant only in regression models where Motivation was excluded.

Finally, this study examined the mediation effects of motivation on the demographic variables. First, concerning the health status variable, Table 4 shows this variable was in fact partially mediated by motivation, (that it would 
somewhat independently affect dietary behavior). To explain this effect, let us first look at equation 5. Here, health status is statistically significant $(\mathrm{t}$-stat $=4.04)$ when it is treated as an independent variable. Next, equation 6 shows that when motivation and knowledge are added as independent variables, the coefficient for health motivation $(-0.111)$ with t-stat (-2.69) has become negative. To clarify this effect, one would say that individuals with lower health status (i.e., higher levels of health problems) engage in healthier dietary behaviors. Then when motivation is added to the equation, all of this positive effect is mediated by motivation so that the overall effect becomes negative. To further affirm that this is a partial mediation one can look at equation 9. Here it could be seen the magnitude of health status increased and became more statistically significant when motivation was regressed on health status as a dependent variable.

The age coefficient also changes when motivation and knowledge are included. This might be indicative of partial mediation. The magnitude reduces from 0.051 to 0.036 whereas the magnitude of the t-stat increases (from 8.92 to 9.43). Among education, income and gender, gender seems to be substantially influenced by the motivation variable in an equation with dietary behaviors as the dependent variable. Gender is also significantly associated with motivation.

The most important implication of this study is that efforts made in the future by government agencies, educators, and private industry, with the goal of affecting the dietary patterns of people, will need to give adequate consideration to motivations driving these choices. Because motivation acts as a mediator to nutrients' dietary functional knowledge, it must be treated as an essential component to any model that maps dietary health behavior. Consistent with previous studies, this paper has shown that educating the public on healthy eating practices is not enough to inspire healthful changes to existing eating patterns. Furthermore, instead of educating the public on general health implications of better diet, efforts should be made to highlight the specific role of nutrients. Food marketers of both fresh produce and processed foods are increasingly using nutrient content claims in advertising. One study found that food ads with nutrient-contents were considered healthier than food ads focusing on taste [24]. Public institutions should also consider nutrition-health campaigns that highlight specific health function of nutrients to effect changes in diet.

Future studies can further develop the findings from this research. First, the level of motivation could, indeed, vary by an individual's health status, i.e., diagnosed with a specific diet-related health problem. For example, those with diabetes might adopt healthy dietary behavior due to their higher risk for health complications. Therefore, this potential correlation should be controlled for in subsequent analyses. Moreover, to avoid survey response biases, future studies would benefit from using revealed dietary behavior data rather than a stated survey response variable. It is also important for future studies to examine the economic impact of this research on policies that are aimed at improving dietary behaviors. Understanding that motivation is a critical component of dietary choices will help policy makers, researchers, and educators allocate funding for research and marketing efforts most effectively and efficiently. Future studies should focus on determining the factors that are driving dietary motivations to improve their dietary behaviors. Ultimately, this will help to guide policies that could dramatically improve peoples' health and reduce the financial burden that dietary-related health conditions impose on the healthcare system.

\section{Appendix}

Table A1. Income Group Classification.

\begin{tabular}{llll}
\hline Group Number & Income Range & Group Number & Income Range \\
\hline 1 & Less than $\$ 5,000$ & 14 & $\$ 65,000-\$ 69,999$ \\
2 & $\$ 5,000-\$ 9,999$ & 15 & $\$ 70,000-\$ 74,999$ \\
3 & $\$ 10,000-\$ 14,999$ & 16 & $\$ 75,000-\$ 79,999$ \\
4 & $\$ 15,000-\$ 19,999$ & 17 & $\$ 80,000-\$ 84,999$ \\
5 & $\$ 20,000-\$ 24,999$ & 18 & $\$ 85,000-\$ 89,999$ \\
6 & $\$ 25,000-\$ 29,999$ & 19 & $\$ 90,000-\$ 94,999$ \\
7 & $\$ 30,000-\$ 34,999$ & 20 & $\$ 95,000-\$ 99,999$ \\
8 & $\$ 35,000-\$ 39,999$ & 21 & $\$ 100,000-\$ 124,99$ \\
9 & $\$ 40,000-\$ 44,999$ & 22 & $\$ 125,000-\$ 149,99$ \\
10 & $\$ 45,000-\$ 49,999$ & 23 & $\$ 150,000-\$ 199,99$ \\
11 & $\$ 50,000-\$ 54,999$ & 24 & $\$ 200,000-\$ 249,99$ \\
12 & $\$ 55,000-\$ 59,999$ & 25 & $\$ 250,000$ or more \\
13 & $\$ 60,000-\$ 64,999$ & & \\
\hline
\end{tabular}

Table A2. Education Group Number Classification.

\begin{tabular}{ll}
\hline Group Number & Education Level \\
\hline 1 & Grade School \\
2 & Some High School \\
3 & Graduated High School \\
4 & Some College \\
5 & Graduated from college - 2 year \\
6 & Graduated from college - 4 year \\
7 & Some Post Graduate \\
8 & Post Graduate Degree \\
11 & Some Technical or Vocational School \\
12 & Completed Technical or Vocational school \\
9 & Other \\
\hline
\end{tabular}

\section{References}

[1] Fardet, A. and Boirie, Y. (2014), Associations between food and beverage groups and major diet-related chronic diseases: an exhaustive review of pooled/meta-analyses and systematic reviews, Nutrition Reviews. Vol. 72, No. 12. [place unknown]: Oxford Academic. p. 741-762.

[2] McCullough, M. L., Feskanich, D., Stampfer, M. J. et al. (2002), Diet quality and major chronic disease risk in men and women: moving toward improved dietary guidance, The American Journal of Clinical Nutrition. Vol. 76, No. 6. [place unknown]: Oxford Academic. p. 1261-1271.

[3] Solbak N. M., Xu J., Vena J. E., Csizmadi I., Whelan H. K., Robson P. J. (2017) Diet quality is associated with reduced incidence of cancer and self-reported chronic disease: Observations from Alberta's Tomorrow Project, Preventive Medicine. Vol. 101. [place unknown]: Elsevier. p. 178-187. 
[4] Schiwal A., Fauth E. B., Wengreen H., Norton M. (2020). The Gray Matters App Targeting Health Behaviors Associated with Alzheimer's Risk: Improvements in Intrinsic Motivation and Impact on Diet Quality and Physical Activity. Vol. 24. [place unknown]: [The Journal of Nutrition, Health \& Aging]. p. 893-899.

[5] Frerichs L., Bess K., Young T. L., Hoover S. M., Calancie L., Wynn M., McFarlin S., Cene C. W., Dave G., Corbie-Smith G. (2020). A Cluster Randomized Trial of a Community-Based Intervention Among African-American Adults: Effects on Dietary and Physical Activity Outcomes. Vol 21. [place unknown]: [Prevention Science]. p. 344-354.

[6] Dashti S., Dabaghi P., Tofangchiha S. (2020). The effectiveness of training program based on protective motivation theory on improving nutritional behaviors and physical activity in military patients with type 2 diabetes mellitus. Vol. 9, No. 7. [place unknown]: [Medknow Publications]. p. 3328-3332.

[7] Wardle, J., Parmenter, K. and Waller, J. (2000), Nutrition knowledge and food intake, Appetite. Vol. 34, No. 3. [place unknown]: Elsevier. p. 269-275.

[8] Brug, J. (2008), Determinants of healthy eating: motivation, abilities and environmental opportunities. Family Practice, Vol. 25, No. Supplement 1. [place unknown]: Oxford University Press., p. 50-55.

[9] De Boer J., Schösler H., Aiking H. (2017) Towards a reduced meat diet: Mindset and motivation of young vegetarians, low, medium and high meat-eaters. Vol. 113. [place unknown]: Appetite. p. 387-397.

[10] Petrovici, D. A. and Ritson, C. (2006), Factors influencing consumer dietary health preventative behaviours, BMC Public Health. Vol. 6, No. 222. [place unknown]: [BioMed Central Ltd].

[11] Moorman, C. and Matulich, E. (1993), A model of consumers' preventive health behaviours: The role of health motivation and health ability, Journal of Consumer Research. Vol. 20, No. 2. [place unknown]: [Oxford Academic]. p. 208-228.

[12] Miller, L. M. S. and Cassady, D. L. (2012), Making healthy food choices using nutrition facts panels. The roles of knowledge, motivation, dietary modifications goals, and age. Appetite, Vol. 59, No. 1. [place unknown]: Academic Press Inc. p. 129-139.

[13] Michaelidou, N., Christodoulides, G. and Torova, K. (2012), Determinants of healthy eating: a cross-national study on motives and barriers. International Journal of Consumer Studies, Vol. 36, No. 1. [place unknown]: [Oxford University Press]. p. 17-22.
[14] Sun, T., Lin, S. and Kolodinsky, J. (2014), Hierarchical trait predictors of healthy diet: a comparison between US and Chinese young consumers, International Journal of Consumer Studies. Vol. 38, No. 6. [place unkown]: Appetite. p. 620-627.

[15] Steptoe, A. and Wardle, J. (1999), Motivational factors as mediators of socioeconomic variations in dietary intake patterns, Psychology and Health. Vol. 14, No 3. [place unknown]: [Taylor \& Francis]. p. 391-402

[16] Povey, R., Conner, M., Sparks, P. et al. (1998), Interpretations of healthy and unhealthy eating, and implications for dietary change. Health Education Research, Vol. 13, No. 2. [place unknown]; Oxford University Press. p. 171-183.

[17] Eikenberry, N. and Smith, C. (2004), Healthful eating: perceptions, motivations, barriers, and promoters in lowincome Minnesota communities, Journal of the American Dietetic Association. Vol. 104, No. 7. [place unknown]: Elsevier p. 1158-61

[18] Hochbaum, G., Rosenstock, I., and Kegels, S. (1952), Health belief model. United States Public Health Service. [Washington, DC.]: SAGE.

[19] Becker, M. H., Maiman, L. A., Kirscht, J. P. et al. (1977), The Health Belief Model and Prediction of Dietary Compliance: A Field Experiment. Journal of Health and Social Behaviour, Vol. 18 No. 4. [place unknown]: American Sociological Association. p. 348-366.

[20] National Institutes of Health (NIH), Office of Dietary Supplements. (2019). Potassium Fact Sheet for Consumers. available at https://ods.od.nih.gov/pdf/factsheets/PotassiumConsumer.pdf (accessed 01 April 2020).

[21] Mayo Clinic. (2016), High cholesterol. Retrieved from $\mathrm{http}$ :/www.mayoclinic.org/diseases-conditions/high-bloodcholesterol/diagnosis-treatment/diagnosis/dxc-20181913

[22] Baron, R. M. and Kenny, D. A. (1986), The moderatormediator variable distinction in social psychological research: Conceptual, strategic, and statistical considerations. Journal of Personality and Social Psychology, Vol. 51 No. 6. [place unknown]: American Phycological Association. p. 1173-1182.

[23] Kenny, D. A., Kashy, D. A., and Bolger, N. (1998), Data analysis in social psychology. Gilbert, D., Fiske, S., and Lindzey, G. (Eds.), The Handbook of Social Psychology

[24] Choi, H., Paek, H-J., and King, K. W. (2012) Are nutrientcontent claims always effective? International Journal of Advertising. Vol. 31, No. 2. [place unknown]: Elsevier., p. 421-443. New York: McGraw-Hill., p. 233-265. 